ments as to how practices may differ in the home than in the hospital. Each patient care practice and procedure must be evaluated on its own merit. For example, tracheostomy care may be different in the home than the hospital because most tracheostomies in hospitals are new wounds. A new wound needs to be treated with sterile aseptic technique, whereas a longterm patient with a tracheostomy probably could have clean technique used without an increased infection risk. Clean technique may include cleaning the inner cannula with soap and water and soaking in $70 \%$ alcohol for 5 minutes followed by a thorough rinsing with normal tap water.

On the other hand, infection control policies for IV care should not be altered because the blood stream is such an opportune media for microbes to grow. The patient is at increased risk of infection in the home as well as the hospital when he is receiving IV fluids. We need to continue changing sites, dressings, and tubing every 48 to 72 hours until studies prove otherwise.

Each home health agency needs to look closely at their practices. It is a good idea for them to consult infection control practitioners when making infection control-related decisions.

Sue Crow, RN, MSN

Nurse Epidemiologist Louisiana State University

Medical Center

Shreveport, Louisiana

\section{Infection Control Measures for the Use of Amniotic Membranes}

\section{To the Editor:}

With the planned opening of a burn unit at our institution, concern has been present over the use of amniotic membranes for coverage of burn wound surfaces. Review of recent studies examining the use of such biologic membranes do not go into great detail discussing infection control-related issues. ${ }^{1,2}$

I would be grateful for advice and/ or references that deal with topics such as risk of transmission of such diseases

as hepatitis B, syphilis, etc. with the use of such products.

\title{
REFERENCES
}

1. Matthews RN, Faulk WP, Bennett JP: A review of the role of amniotic membranes in surgical practice. Obstet Gynecol Amnu 1982; 11:31-58.

2. Jasinkowski NL. Cullum JL: Human amniotic membrane. AORN J 1984; 38(5):894-899.

\section{Larry M. Baddour, MD \\ Hospital Epidemiologist Infection Control Department Regional Medical Center at Memphis Memphis, Tennessee}

William M. Valenti, MD and Florence Jacoby, RN of Strong Memorial Hospital, Rochester, New York, respond to Dr. Baddour's query.

Amniotic membranes have proven useful as temporary biologic dressings on wounds prior to grafting. In full thickness injuries, the membranes have gained immediate adherence and have decreased pain and bacterial contamination of wounds.

If these membranes are procured, stored and used appropriately, they can prove life saving for patients with full thickness wounds both thermal and nonthermal. We are not aware of any reports of disease transmission associated with amniotic membranes. However, as with any material donated from humans, the potential for transmission of infection is present and the infection control policy for the burn unit should attempt to minimize the risk to recipients. Robson et al used fresh amniotic membranes obtained from "seronegative" mothers who had no history of either premature rupture of the membranes or endometritis. ${ }^{1}$ Although the authors do not define seronegative, we suggest that the donors have negative serologies for syphilis and hepatitis B surface antigen. According to most protocols for amniotic membranes, the material is cultured at the time of procurement and before use. In addition, Robson did not use material after 6 weeks of storage. The donor should also be in good health without any acute or chronic underlying disease or history of drug or substance abuse. As testing for the human T-cell lymphotropic virus (HTLV-III) becomes more specific and more widely available, protocols for organ transplant may include this type of screening as well.

The policy should include details regarding procurement, storage and processing of membranes as well as guidelines for acceptable donors. If certain aspects of the procedure are planned in advance, the use of amniotic membranes should carry a low risk of infection to the recipient.

\section{REFERENCE}

1. Robson MC, et al: Amniotic membranes as a temporary wound dressing. Surg Gynecol Obstet, 1973; 136:904-906.

William M. Valenti, MD Hospital Epidemiologist Florence Jacoby, RN Burn Nurse Clinician Strong Memorial Hospital Rochester, New York

\section{Use of Personal Items Could Present Problems}

\section{To the Editor:}

A patient who had been hospitalized at our institution for several months developed new respiratory symptoms suggestive of an allergic pneumonitis. The patient unknown to the staff had recently begun using in the hospital her personal humidifier brought from her home. Culture of the humidifier (spout, mist, tank, water, etc.) revealed multiple species of bacteria usually associated with water or hospital surfaces-Flavobacterium sp., Pseudomonas sp., Bacillus sp., and staphylococci. In addition, cultures of the mist and several other sites of the humidifier grew Aspergillus species. The patient's symptoms ended when she discontinued use of the humidifier.

In light of this incident it is important to remember that items patients bring into the hospital for personal use could be a potential source of contamination that would not be anticipated. Humidifiers can be a source of bacteria or fungi. Hospital staff should be alerted to the dangers of these devices which patients may elect to use at their own discretion.

Helen R. Bopp, MS Environmental Control Specialist Harold C. Neu, MD Hospital Epidemiologist Columbia-Presbyterian Medical Center New York, New York 